\title{
MEDIDA DE SELETIVIDADE DOS CURSOS DE ENGENHARIA DA UFF COM MODELOS DEA
}

\author{
Maria Helena Campos Soares de Mello \\ João Carlos C. B. Soares de Mello \\ Lidia Angulo-Meza ${ }^{3}$
}

\begin{abstract}
Resumo: A Universidade Federal Fluminense possui atualmente, quatorze Cursos de Engenharia em três Campi distintos. Embora os alunos tenham realizado o mesmo concurso de admissáo (vestibular), foram admitidos nos cursos com notas diferentes, já que as notas mínimas para ingresso em cada um dos cursos foram diferentes. Isso significa que os calouros têm níveis de conhecimento diferentes e apresentam dificuldades diferentes ao longo dos seus estudos no primeiro período. Uma das razóes que se aponta para a diferença entre os conhecimentos dos estudantes é a quantidade de candidatos para os cursos. Muitas outras razões podem ser apontadas para explicar o desempenho dos estudantes ao longo do curso. Neste trabalho, pretende-se desenvolver um índice de desempenho, usando DEA (Data Envelopment Analysis) no qual, cada curso é avaliado de acordo com a capacidade de atrair bons alunos, que será denominada de seletividade. Utilizou-se um modelo DEA-BCC (Banker, Charnes e Cooper), também conhecido como DEA-VRS (Retornos Variáveis de Escala). Foram utilizados um input e dois outputs. O único input é a relação candidato/vaga e os outputs são as notas de corte em cada fase do vestibular. Devido à maior importância da nota final é introduzida uma restrição do tipo cone-ratio.
\end{abstract}

Palavras Chave: DEA, desempenho de estudantes, avaliação de cursos

\begin{abstract}
The Fluminense Federal University has at present, fourteen Engineering Courses in three different Campi. Though the pupils have carried out the same admission exam, they were admitted in the courses with different marks. That means that the freshmen have different knowledge levels and present different difficulties along his studies in the first period. One of the reasons what one points for the difference between the students knowledges is the quantity of candidates for each course. Many other reasons can be pointed to explain the students performance along the course. In this work, we develop a performance measure using DEA (Date Envelopment Analysis) in which, each course is valued in accordance with the capacity of attracting good pupils. Such a measure will be called selectivity. For that we used a DEA-BCC (Banker, Charnes and Cooper) model, also known as DEA-VRS (Variable Returns to Scale). An input and two outputs were used. The only input is the relation candidate/place and the outputs are the cut marks in each phase of the exam. Due to the biggest importance of the final mark we introduced a cone ratio restriction.
\end{abstract}

Key words: DEA, students' performance, evaluation of courses

\footnotetext{
Universidade Federal Fluminense, e-mail: mhelena@id.uff.br

Universidade Federal Fluminense, e-mail: jccbsmello@id.uff.br

Universidade Federal Fluminense, e-mail: lidia_a_meza@pq.cnpq.br
} 


\section{INTRODUÇÃO}

Dentro da atual política de aumento de vagas no ensino superior, o Rio de Janeiro é um estado privilegiado no que concerne a universidades públicas. Em especial em relação à oferta de vagas para os cursos de Engenharia, onde quase todas atuam em mais de um campus. Se, para a população, isso é um fator extremamente positivo, levanta problemas gerenciais para as instituiçôes. A oferta total de vagas pode variar abruptamente em função de uma decisão tomada pelas outras instituiçóes, o que compromete a estabilidade da relação entre oferta e procura. Mesmo sem levar em conta as outras instituições, podem existir variaçôes bruscas de demanda, devido à tendência de certos alunos em procurarem cursos onde acreditam ter mais facilidade em serem aprovados, como mostrado por Soares de Mello et al. (2003b). Estas variações na relação entre oferta e demanda podem provocar mudanças no nível de entrada e consequentemente no aproveitamento dos alunos no curso. Estudos sobre esta mudança no aproveitamento do curso podem ser encontrados em Soares de Mello et al (2004) e em Silva e Padoin (2008).

Outro fato que afeta o nível dos alunos é que muitos desistem do curso para onde foram aprovados em virtude de terem sido aprovados em outro(s) curso(s) semelhante(s) em outras instituições de ensino. Estes fatores agravam o problema da evasão, já de si grave em alguns dos cursos de Engenharia (Moreira da Silva et al. 2006).

Acredita-se dentro da Universidade que, quanto maior a relaçáo candidato/vaga de um curso melhores serão os alunos desse curso. Pretende-se neste artigo verificar a validade dessa suposição e analisar quais cursos conseguem atrair alunos com melhor desempenho, para relaçóes candidat/ vaga semelhantes. Para isso será usado um modelo de Análise de Envoltória de Dados (DEA com restrição de convexidade, o chamado modelo BCC (Banker et al. 1984)

$\mathrm{Na}$ próxima seção serão apresentados os fundamentos de DEA e uma breve revisão da sua aplicação em educação. Na seção 3 serão apresentados a interiorizaçáo da UFF e o seu vestibular. $\mathrm{Na}$ seção 4 são apresentados o modelo usado e os principais resultados. Finalmente, na seção 5, algumas conclusóes serão apresentadas e idéias sobre trabalhos futuros.

\section{ANÁLISE ENVOLTÓRIA DE DADOS - DEA}

A Análise Envoltória de Dados - DEA é uma técnica não paramétrica de medida de eficiência. Ela realiza avaliaçáo de unidades produtivas, chamadas unidades tomadoras de decisão (Decision Making Units - DMUs). Aqui o termo produtivo é usado de forma ampla, e não está necessariamente relacionado a nenhum tipo de produção física. DEA é especialmente indicado quando os aspectos financeiros forem difíceis de medir ou quando não haja interesse em levá-los em conta (Soares de Mello et al. 2003a). Esta abordagem otimiza o quociente entre a soma ponderada dos produtos (outputs) e a soma ponderada dos recursos (inputs). Para cada DMU a ponderação é feita com pesos diferentes, de forma que cada uma dê mais valor aos fatores em que tem melhor desempenho.

O modelo CCR é apresentado em (1). Neste modelo Eff é a eficiência da DMU $o$ em análise; $v_{i}$ e $u_{j}$ são os pesos de inputs $i, i=1, \ldots, r$, e outputs $j, j=1, \ldots, s$ respectivamente; $x_{i k}$ e $y_{j k}$ são os inputs $i$ e outputs $j$ da DMU $k, k=1, \ldots, n ; x_{i o}$ e $y_{j o}$ são os inputs $i$ e outputs $j$ da DMU $o$.

$\operatorname{Max} \operatorname{Eff}_{o}=\square_{j=1}^{s} u_{j} y_{j o}$

sujeito a

$\square_{i=1}^{r} v_{i} x_{i o}=1$

$\square_{j=1}^{s} u_{j} y_{j k} \square \square_{i=1}^{r} v_{i} x_{i k} \square 0, \square k$

$v_{i}, u_{j} \square 0, \square i, j$

Detalhes matemáticos sobre a modelagem DEA podem ser vistos, por exemplo, em Cooper et al. (2000) ou em Soares de Mello et al (2005).

Como cada DMU escolhe os pesos que maximizam o seu desempenho, DEA é considerada uma técnica benevolente. É comum, principalmente quando o número de DMUs é pequeno, que haja uma grande quantidade de empates entre as unidades eficientes, ou seja, aquelas cuja eficiência é unitária, a máxima eficiência. Isto deve-se à estrutura matemática dos modelos DEA que permite que uma DMU seja considerada eficiente com vários conjuntos de pesos. Em particular, podem ser atribuídos pesos zeros a algum input ou output, o que significa que essa variável foi descartada na avaliação. Para contornar este problema 
existem métodos clássicos de aumento de discriminação em DEA (Angulo-Meza and Lins 2002).

A Análise Envoltória de Dados tem sido largamente utilizada em avaliação educacional. $\mathrm{O}$ primeiro artigo sobre o tema (Charnes et al. 1978) já tratava da avaliação de escolas. Uma extensa revisão sobre o uso de DEA em avaliação educacional pode ser encontrada em (Soares de Mello et al. 2006). Neste trabalho será feita apenas uma breve revisão sobre a aplicação de DEA em vestibulares e processos semelhantes.

Sarrico et al. (1997) usam DEA para averiguar quais alunos são mais apropriados para entrarem numa universidade britânica. Já Soares de Mello et al.(2001) usam DEA para avaliar a eficiência de cada município fluminense em conseguir que os seus alunos tenham acesso ao ensino superior. Eles avaliam também o sucesso da UFF em divulgar os seu vestibular nesses municípios. Usando modelos DEA-GSZ de Lins et al (2003), o trabalho posterior de Gomes et al (2005) usa os resultados da avaliaçáo da influencia do vestibular da UFF nos municípios do interior fluminense de Soares de Mello et al. (2001) para verificar quantos alunos cada município fluminense deveria conseguir aprovar para os cursos da UFF num determinado vestibular. Finalmente, Soares de Mello et al (2002b) usam resultados do vestibular da UFF como variável de entrada num modelo DEA que mede a eficiência de turmas de Cálculo I.

\section{O VESTIBULAR DA UFF}

\section{A UFF NO INTERIOR}

A UFF atualmente dispóe de cursos de Engenharia em três campi: Niterói, Volta Redonda e Rio das Ostras, três cidades do Estado do Rio de Janeiro. Niterói é a sede e nas outras duas funcionam pólos de interiorização. Sobre a fundação dos cursos de engenharia da UFF ver Cantanhede (2002). Trabalhos anteriores já abordaram o tema da interiorizaçáo da UFF. Soares de Mello et al (2002a) avaliaram o impacto da presença da UFF em todos os municípios onde ela tem uma atuação usando o método multicritério MACBETH de Bana e Costa e Vansnick (1995).

Com o intuito de aprimorar esta avaliação, Rangel et al (2003) avaliaram o impacto apenas nos municípios onde existe cursos de graduação da UFF. Para tal usaram uma variante do método
UTA de Jacquet-Lagreze e Siskos (1982) com os pesos limitados pelo método MACBETH. Além do impacto da UFF em cada município, determinaram a função de utilidade de cada critério.

Até o final do século XX a maioria dos programas de interiorizaçáo da UFF não envolvia os cursos de engenharia, a exceção era o curso de Engenharia Industrial Metalúrgica oferecida na cidade de Volta Redonda. Quando os cursos de engenharia começaram a participar da interiorização Vaz et al (2002) fizeram uma análise qualitativo dos vários modelos de interiorizaçáo discorrendo quais seriam mais adequados para os cursos de engenharia.

Dada a grande dinâmica do processo de interiorização há uma necessidade constante de atualizar as avaliaçóes, Soares de Mello (2005) usa métodos ordinais multicritério para verificar essa evolução.

Finalmente, com a introdução do ensino a distancia na UFF através do consorcio CEDERJ foi necessário avaliar o impacto dos vários pólos deste consorcio. Isto foi feito por Gomes Jr et al (2008) usando o método ordinal de Copeland.

\section{O MODELO DO VESTIBULAR}

O vestibular é unificado, isto é, é o mesmo para todos os cursos de graduação presenciais da UFF. Este é realizado em duas etapas. A primeira, consiste de uma prova de múltipla escolha, de caráter eliminatório. Para ser aprovado nesta fase, em princípio, o candidato precisa obter pontuaçáo de pelo menos $50 \%$ do total. No entanto, este percentual pode ser aumentado ou diminuído para alguns cursos. Esta alteração depende do número de candidatos que obtém um total de acertos superior a $50 \%$ e depende também do número de vagas oferecidas por cada curso. Caso a divisão entre o número de alunos com acertos superiores a $50 \%$ e o total de vagas oferecida pelo curso seja a inferior a três, são aprovados na primeira fase alunos com total de acertos inferior a 50\%, até a referida divisão ficar igual a três. Pelo contrário, se essa divisão for superior a oito, são eliminados candidatos com acertos superiores a 50\% de forma a que a divisão fique igual a oito. Para mais detalhes jurídicos e operacionais sobre o vestibular da UFF ver COSEAC (2007).

A segunda etapa do vestibular é específica para cada curso, ou grupo de cursos. No caso das 
Engenharias (excetuando-se Engenharia Química) é composta por uma redação em língua portuguesa e uma prova discursiva de Matemática e Física. Esta etapa é classificatória. Após a divulgação das notas, os candidatos são convocados para a matrícula por ordem de notas. Caso náo compareçam, são convocados os seguintes em ordem de nota final. Este processo é chamado de "reclassificação" e são realizadas tantas reclassificações quantas necessário para se preencher a quantidade de vagas oferecidas, desde que existam candidatos disponíveis.

\section{MODELAGEM}

Neste artigo, os cursos de engenharia da UFF são as DMUs. Foi excluído o curso de Engenharia Química uma vez que sua segunda prova é diferente dos outros cursos de engenharia. Desta forma garante-se a homogeneidade das DMUs. Se essa homogeneidade não fosse garantida, o modelo perderia validade por ter incorrido numa "armadilha de DEA", tal como referido por Dyson et al.(2001). As variáveis consideradas nesta análise são como input a relaçáo candidato/vaga de cada curso de Engenharia da UFF no vestibular de 2008; como outputs o total de acertos do último aprovado na primeira etapa do vestibular em cada curso e o total de pontos do último aprovado para cada curso, ao final da segunda fase. Os dados para os treze cursos analisados estáo apresentados na Tabela 1.

Esta seleção de variáveis apresenta um pequeno problema. Como mencionado na descrição do vestibular da UFF existe uma nota de corte na primeira fase, que é $50 \%$ de acertos. No entanto, como também foi mencionado, esta nota não se aplica para cursos com poucos ou com muitos aprovados. Ou seja, a distorção existe mas não é muito grande. Assim o objetivo do modelo é avaliar se os alunos ingressantes em cada curso tiveram notas compatíveis com a relação candidato/ vaga do curso.

Foi escolhido o modelo BCC devido ao fato do input ser uma razáo e os outputs serem limitados (Hollingsworth and Smith 2003). Foi escolhida a orientação a outputs.

A Tabela 2 apresenta os resultados da eficiência obtidos com o software SIAD (Angulo-Meza et al. 2005).

Tabela 1. DMUs e seus dados.

\begin{tabular}{|c|c|c|c|}
\hline DMU & Candidato/vaga & Nota 1 & Nota 2 \\
\hline Agrícola & 2,75 & 16 & 25,85 \\
\hline Civil & 10,01 & 33 & 53,08 \\
\hline Agronegócios (Volta Redonda) & 2,55 & 14 & 22,15 \\
\hline Petróleo & 18,07 & 33 & 66,11 \\
\hline Produção (Niterói) & 13,35 & 33 & 71,01 \\
\hline Produção (Rio das Ostras) & 6,30 & 27 & 43,26 \\
\hline Produção (Volta Redonda) & 6,08 & 30 & 48,24 \\
\hline Telecomunicaçóes & 5,01 & 28 & 48,16 \\
\hline Elétrica & 8,09 & 33 & 56,20 \\
\hline Mecânica (Niterói) & 12,62 & 33 & 63,56 \\
\hline Mecânica (Volta Redonda) & 5,19 & 26 & 42,97 \\
\hline Metalúrgica (Volta Redonda) & 3,09 & 14 & 30,64 \\
\hline Meio Ambiente & 9,31 & 33 & 53,71 \\
\hline
\end{tabular}

Fonte: COSEAC 
Tabela 2. Eficiências para os cursos avaliados

\begin{tabular}{|c|c|}
\hline DMU & Eficiência \\
\hline Agrícola & 100,00 \\
\hline Civil & 100,00 \\
\hline Agronegócios (Volta Redonda) & 100,00 \\
\hline Petróleo & 100,00 \\
\hline Produção (Niterói) & 100,00 \\
\hline Produção (Rio das Ostras) & 89,03 \\
\hline Produção (Volta Redonda) & 100,00 \\
\hline Telecomunicações & 100,00 \\
\hline Elétrica & 100,00 \\
\hline Mecânica (Niterói) & 100,00 \\
\hline Mecânica (Volta Redonda) & 91,75 \\
\hline Metalúrgica (Volta Redonda) & 100,00 \\
\hline Meio Ambiente & 100,00 \\
\hline
\end{tabular}

Observa-se que quase todos os cursos foram eficientes, o que indica que existe uma forte relação (não linear) entre a relação candidato/vaga e os alunos de vestibular. Observa-se também que dois cursos náo foram eficientes que pode ser explicado pelo desempenho dos alunos ser abaixo do que a relaçáo candidato/vaga faria esperar. Esse dois cursos estão situados fora da sede e há cursos semelhantes na sede de Niterói.

Dado que existem muitos cursos 100,00\% eficientes, a quantidade de informação obtida da tabela é muito limitada. Para aprimorar as conclusóes que podem ser obtidas do modelo, promoveu-se um desempate entre algumas das DMUs. Para esse efeito foi utilizada a técnica das regióes de segurança (Allen et al. 1997). Impôs-se que a nota final deve ter uma importância maior que a nota de corte da primeira fase. Os resultados com essa restrição são apresentados na Tabela 3.

Da tabela 2 pode-se verificar que quando um curso é sediado em mais de uma cidade, o sediado em Niterói é mais seletivo que o com sede no interior. Quando foi estudada a atratividade a conclusão era exatamente a oposta (Soares de Mello et al. 2008).
Tabela 3. Eficiência dos cursos com restrição aos pesos

\begin{tabular}{|c|c|}
\hline DMU & Eficiência (\%) \\
\hline Agrícola & 100,00 \\
\hline Agronegócios (Volta Redonda) & 100,00 \\
\hline Produção (Niterói) & 100,00 \\
\hline Telecomunicaçóes & 100,00 \\
\hline Elétrica (Niterói) & 100,00 \\
\hline Metalúrgica (Volta Redonda) & 100,00 \\
\hline Produção (Volta Redonda) & 98,13 \\
\hline Petróleo & 96,55 \\
\hline Mecânica (Niterói) & 96,15 \\
\hline Meioambiente & 95,46 \\
\hline Civil & 93,57 \\
\hline Mecânica (Volta Redonda) & 90,33 \\
\hline Produção (Rio das Ostras) & 87,16 \\
\hline
\end{tabular}

\section{CONCLUSÓES}

A principal conclusão deste trabalho é validar o senso comum de que as maiores relaçóes candidato/vaga tendem a propiciar alunos com melhor desempenho. Verificou-se no modelo sem restriçóes aos pesos que apenas dois cursos com sede no interior não foram eficientes, ou seja, os alunos classificados tiveram desempenho inferior à relação candidato/vaga do curso faria supor. Isto levanta a hipótese que os alunos escolheram esses cursos náo por interesse mas para tentar o vestibular mais fácil. O resultado obtido com restriçóes aos pesos aponta na mesma direção. Esta hipótese é consistente com a oscilação da procura verificada em Soares de Mello et al (2003b).

Como desenvolvimento futuro, pretende-se fazer uma avaliação semelhante para cursos de outras áreas.

\section{BIBLIOGRAFIA}

Allen, R., A. Athanassopoulos, R.G. Dyson and E. Thanassoulis. 1997. "Weights restrictions and value judgements in data envelopment analysis: evolution, development and future directions." Annals of Operations Research 73:13-34. 
Angulo-Meza, Lidia, Luiz Biondi Neto, João Carlos Correia Baptista Soares de Mello and Eliane Gonçalves Gomes. 2005. "ISYDS - Integrated System for Decision Support (SIAD Sistema Integrado de Apoio a Decisão): A Software Package for Data Envelopment Analysis Model." Pesquisa Operacional 25(3):493-503.

Angulo-Meza, Lidia and Marcos Pereira Estellita Lins. 2002. "Review of methods for increasing discrimination in data envelopment analysis." Annals of Operations Research 116:225-242.

Bana e Costa, C.A. and J.C. Vansnick. 1995. "Uma nova abordagem ao problema da construção de uma função de valor cardinal: MACBETH." Investigação Operacional 15:15-35.

Banker, R.D., A. Charnes and William W. Cooper. 1984. "Some models for estimating technical scale inefficiencies in data envelopment analysis." Management Science 30(9):1078-1092.

Cantanhede, Otavio. 2002. A Escola Fluminense de Engenharia: Sua criação e sua obra 1952/2002. Niterói: EdUFF.

Charnes, A., W.W. Cooper and E. Rhodes. 1978. "Measuring the efficiency of decision-making units." European Journal of Operational Research 2:429-444.

Cooper, William W., L. Seiford and K. Tone. 2000. Data envelopment analysis: A comprehensive text with models, applications, references and DEA-solver software. Boston: Kluwer.

COSEAC. 2007. "Edital do Vestibular UFF 2008." Niterói: Universidade Federal Fluminense.

Dyson, R.G., R. Allen, Ana S. Camanho, V.V. Podinovski, C.S. Sarrico and E.A. Shale. 2001. "Pitfalls and protocols in DEA." European Journal of Operational Research 132(2):245-259.

Gomes, Eliane Gonçalves, João Carlos Correia Baptista Soares de Mello and Marcos Pereira Estellita Lins. 2005. "Uniformização da fronteira eficiente em modelos de análise de envoltória de dados com ganhos de soma zero e retor- nos constantes de escala." Pesquisa Operacional 25(2):261-277.

Gomes Junior, Silvio F., João Carlos Correia Baptista Soares de Mello and Maria Helena Campos Soares de Mello. 2008. "Utilização do método de Copeland para avaliação dos pólos regionais do CEDERJ." Rio's international journal on sciences of industrial and systems engineering and management 2(4):87-98.

Hollingsworth, B. and P. Smith. 2003. "Use of ratios in data envelopment analysis." Applied Economics Letters 10(11):733-735.

Jacquet-Lagreze, E. and J. Siskos. 1982. "Assessing a set of Additive Utility Functions for Multicriteria Decision-Making, the UTA Method." European Journal of Operational Research 10(2):151-164.

Lins, Marcos Pereira Estellita, Eliane Gonçalves Gomes, João Carlos Correia Baptista Soares de Mello and Adelino José R. Soares de Mello. 2003. "Olympic ranking based on a zero sum gains DEA model." European Journal of Operational Research 148:312-322.

Moreira da Silva, Rosenir Rita de Cássia, Fernando Benedicto Mainier and Fábio Barboza Passos. 2006. "A contribuição da disciplina de introdução à engenharia química no diagnóstico da evasão." Ensaio: Avaliação e Políticas Públicas em Educação 14(51):261-277.

Rangel, Luiz Alberto Duncan, João Carlos Correia Baptista Soares de Mello, Eliane Gonçalves Gomes and Luiz Flávio Autran Monteiro Gomes. 2003. "Avaliação da interiorizaçáo dos cursos da Universidade Federal Fluminense com o uso conjugado dos métodos UTA e MACBETH." Investigação Operacional 23(1):49-69.

Sarrico, C.S., S.M. Hogan, R.G. Dyson and A. Athanassopoulos. 1997. "Using DEA for planning in UK universities - an institutional perspective." Journal of the Operational Research Society 51(7):789-800.

Silva, Maelin and Maristela Jorge Padoin. 2008. "Relação entre o desempenho no vestibular e o 
desempenho durante o curso de graduação " Ensaio: Avaliação e Políticas Públicas em Educação 16(58):77-94.

Soares de Mello, João Carlos Correia Baptista, Lidia Angulo-Meza, Eliane Gonçalves Gomes, Bruno Pessoa Serapião and M.P.E. Lins. 2003a. "Análise de envoltória de dados no estudo da eficiência e dos benchmarks para companhias aéreas brasileiras." Pesquisa Operacional 23(2):325345.

Soares de Mello, João Carlos Correia Baptista, Eliane Gonçalves Gomes, Lidia Angulo-Meza, Maria Helena Campos Soares de Mello and Adelino José R. Soares de Mello. 2006. "Engineering Post-Graduate Programmes: A Quality and Productivity Analysis." Studies in Educational Evaluation 32:136-152.

Soares de Mello, João Carlos Correia Baptista, Eliane Gonçalves Gomes and Marcos Pereira Estellita Lins. 2002a. "Análise multicritério da presença da Universidade Federal Fluminense com o uso do método MACBETH." Produçáo 11(2):53-67.

Soares de Mello, João Carlos Correia Baptista, Eliane Gonçalves Gomes, Marcos Pereira Estellita Lins and Luiz Alberto Maron Vieira. 2001. "Um caso de estudo de integração SIG-DEA-MCDA: a influência de uma instituição de ensino superior em vários munícipios do estado do Rio de Janeiro." Investigação Operacional 21(2):171-190.

Soares de Mello, João Carlos Correia Baptista, Marcos Pereira Estellita Lins, Maria Helena Campos Soares de Mello and Eliane Gonçalves
Gomes. 2002b. "Evaluating the performance of Calculus classes using operational research tools." European Journal Of Engineering Education 27(2):209-218

Soares de Mello, Maria Helena Campos. 2005. "Avaliação do impacto da interiorização da UFF utilizando métodos ordinais multicritério." In Simpósio Brasileiro de Pesquisa Operacional. Gramado.

Soares de Mello, Maria Helena Campos, Heitor Luiz Murat de Meirelles Quintella and João Carlos Correia Baptista Soares de Mello. 2004. "Avaliação do desempenho de alunos considerando classificaçóes obtidas e opinióes dos docentes." Investigação Operacional 24(2):187-196.

Soares de Mello, Maria Helena Campos, João Carlos Correia Baptista Soares de Mello and Lidia Angulo-Meza. 2008. "Estudo comparado da atratividade de cursos de Engenharia da UFF." In XXXVI Congresso Brasileiro de Educação em Engenharia. São Paulo: ABENGE.

Soares de Mello, Maria Helena Campos, João Carlos Correia Baptista Soares de Mello, F.R. Leta, Artur José Fernandes and Marcos da Rocha Vaz. 2003b. "Análise sobre o momento de opção do aluno por uma modalidade de engenharia." Revista de Ensino de Engenharia 22(1):1-5.

Vaz, Marcos da Rocha, João Carlos Correia Baptista Soares de Mello, Maria Helena Campos Soares de Mello and Heitor Luiz Soares de Moura. 2002. "Participação das engenharias na interiorização dos cursos da UFF." In Congresso Brasileiro de Ensino de Engenharia. Piracicaba. 